\title{
REEXAMINANDO LA DEFINICIÓN DE TRATA DE SERES HUMANOS DEL PROTOCOLO DE PALERMO: LA TRATA COMO FORMA DE EXPLOTACIÓN
}

\author{
Unwrapping the Human Trafficking Definition of the \\ Palermo Protocol: Trafficking as Harmful Exploitation
}

\author{
Ana B. Valverde Cano \\ Doctoranda en Derecho penal \\ Universidad de Granada
}

http://dx.doi.org/10.18543/ed-67(2)-2019pp15-29

Recibido: 30.09.2019

Aceptado: 21.11.2019

\section{Resumen}

Tras el transcurso de casi dos décadas desde la aprobación del Protocolo de Palermo, estamos en condiciones evaluar críticamente si se ha consolidado un régimen transnacional eficaz que permita la asistencia mutua eficaz en materia penal o si, por el contrario, continúa tratándose de un régimen fragmentado. A pesar de la armonización del concepto de trata, una defectuosa comprensión de su naturaleza abusiva ha dado lugar a lecturas muy singulares del fenómeno reflejadas en las normativas domésticas, por ejemplo, estableciendo un delito de trata donde no se exige el empleo de ciertos medios comisivos. El objetivo de este trabajo consiste en ofrecer una reformulación del paradigma de la trata, poniendo el foco en lo que convierte la trata en una práctica abusiva o «de explotación» per se: la utilización de ciertos medios y no únicamente las finalidades de explotación. Este cambio de paradigma, además de ser útil para acometer los desafíos que plantea la cooperación interestatal, nos ayuda a identificar las conductas relevantes en términos de explotación y permite abordar las críticas de los que -en ocasiones, acertadamente- ven en el régimen contra la trata una herramienta de control migratorio más. 


\section{Palabras clave}

Trata de seres humanos. Explotación. Protocolo de Palermo. Cooperación interestatal.

\section{Abstract}

Almost two decades after the adoption of the Palermo Protocol, we are now able to critically assess whether an effective transnational regime has been consolidated to enable effective mutual assistance in criminal matters or whether, on the contrary, it remains a fragmented regime. Despite the harmonisation of the concept of human trafficking, a flawed understanding of its exploitative nature has led to very unique readings reflected in domestic regulations, for example by establishing a trafficking offence where the use of certain means of commission is not required. The aim of this paper is to reformulate the trafficking paradigm, focusing on what makes trafficking an abusive or exploitative practice per se: the use of certain means and not just the purposes of exploitation. This paradigm shift is useful for tackling the challenges posed by inter-state cooperation. Furthermore, it helps us to identify the relevant behaviours in terms of exploitation and allows us to address the criticisms of those who -sometimes rightly- see the anti-trafficking regime as yet another migration control tool.

\section{Keywords}

Human Trafficking. Human Exploitation. Palermo Protocol. International Cooperation. 


\begin{abstract}
SuMARIO: InTRODUCCIÓn. 1. La trata como práctica de explotación. 1.1. ¿Por qué es necesario la redefinición del paradigma desde el que se aborda la trata de personas?. a. La trata de seres humanos: una definición imprecisa. b. Incorporación del delito de trata en las legislaciones nacionales: ¿estamos hablando de lo mismo?. c. La fragmentación del régimen contra la trata: ¿cuáles son las consecuencias?. d. La trata desde el paradigma de la explotación. II. CONCLUSIÓN. BIBLIOGRAFÍA.
\end{abstract}

\title{
I. INTRODUCCIÓN
}

Desde la década de 1990, la lucha internacional contra la trata de personas se ha visto notablemente intensificada y acompañada de una importante acción normativa e institucional (Milivojevic y Pickering 2013, 585-586; Atak y Simeon 2014, 1020; Pérez Alonso 2015, 1147-1194. Sobre el papel de los informes sobre trata de personas -TIP Reports - del Departamento de Estado de los Estados Unidos en la intensificación del régimen anti-trata: Kelley 2017, 12; Taylor e Isgro 2018, 48-60; Villacampa Estiarte 2011, 269-274).

En el año 2000, la Asamblea General de Naciones Unidas adoptó la Convención de las Naciones Unidas contra la Delincuencia Organizada Transnacional. Junto a esta Convención, se aprobaron otros tres Protocolos -instrumentos adicionales que complementan el instrumento principal- que se referían a la trata de personas, el tráfico de inmigrantes y el tráfico ilícito de armas de fuego. El Protocolo de Naciones Unidas para Prevenir, Reprimir y Sancionar la Trata de Personas, Especialmente Mujeres y Niños (que se conoce como Protocolo de Palermo) tiene el propósito de fomentar la cooperación estatal para «prevenir y combatir eficazmente la trata de personas», y supone un hito en la consolidación de una definición internacional de trata de seres humanos (Kemp, 2001, 166; Heintze y Lülf 2016, 154-155).

Hasta entonces, el término había aparecido en diversos acuerdos internacionales durante el siglo XX, fundamentalmente vinculados a la «trata de blancas» o a la trata con fines de explotación sexual. No obstante, no había podido definirse adecuadamente por las importantes discrepancias sobre qué actos constitutivos debían quedar comprendidos en el ámbito de la trata, cuál era su verdadera naturaleza y otras cuestiones conexas, como su relación con la migración ilegal (Irwin 1996; Doezema 1998, 34-50; Grittner 1990; Obokata 2006, 13-17; Gallagher 2010, 12; Limoncelli,2010, 46; Iglesias Skulj 2013, 56-57; Maqueda Abreu 2009, 9-13; Maqueda Abreu 2018, 1251 1253). Por ello, tras explicitar en el Preámbulo que «no hay ningún instrumento universal que aborde todos los aspectos de la trata de personas», el Protocolo de Palermo viene a colmar esta laguna. 
Uno de los aspectos más relevantes del Protocolo de Palermo es su alto número de ratificaciones (174), que lo convierten en una herramienta privilegiada para la creación de un régimen transnacional efectivo contra la trata de personas (Rijken 2013, 17). No obstante, la indefinición del concepto ha supuesto que casi cualquier actividad pueda considerarse abusiva y encajar en el «propósito de explotación» de la trata, lo que cuestiona la propia eficacia del régimen transnacional. Esto puede deberse a que el foco se ha centrado, en gran medida, en el último de los elementos que conforman la definición internacional de la trata -el propósito de explotación-, y no tanto en los elementos que limitan la libertad de elección y que colocan a la persona en una posición en la que puede ser explotada (Allain 2019, 3-5).

El objetivo de este trabajo es ofrecer una reformulación del paradigma de la trata de personas, explorando la verdadera naturaleza de este fenómeno, para facilitar la consecución del objetivo de cooperación interestatal del Protocolo de Palermo. Para ello, en primer lugar, se identificarán los principales desafíos que plantea la falta de seguridad jurídica, por ejemplo, con relación a la institución de la extradición y el principio de doble incriminación penal. En segundo lugar, se argumentará que la naturaleza misma de la trata como práctica de explotación está determinada por el uso de ciertos medios (coercitivos, engañosos, etc.), y no únicamente por el propósito de explotación.

\section{La trata como práctica de explotación}

\section{1. ¿Por qué es necesario la redefinición del paradigma desde el que se aborda la trata de personas?}

\section{a. La trata de seres humanos: una definición imprecisa}

La trata se define en el artículo 3 del Protocolo de Palermo como un fenómeno que incluye tres elementos: en primer lugar, una serie de acciones relacionadas con el movimiento de personas, que permiten incriminar a distintos actores dentro de un sistema complejo (captación, transporte, traslado, acogida o recepción); en segundo lugar, el recurso a mecanismos que implican coacción y/o el abuso de poder, que limitan la libertad de elección (amenaza o uso de la fuerza u otras formas de coacción, rapto, fraude, engaño, abuso de poder o de una situación de vulnerabilidad, o la concesión o recepción de pagos o beneficios para obtener el consentimiento de una persona que tenga autoridad sobre otra); y en tercer lugar, el «propósito de explotación». A continuación, indica que «esa explotación incluirá, como mínimo, la explotación de la prostitución ajena u otras formas de explotación sexual, los trabajos o servicios forzados, la esclavitud o las prácticas análogas a la esclavitud, la servidumbre o la extracción de órganos» (análisis de la definición del 
Protocolo de Palermo en: Pérez Alonso 2008, 173-188; Gallagher 2010, 25-42; y de forma más general, en: Rodríguez López 2016).

Este delito presenta algunas particularidades cuando la víctima es menor de edad, pero por ahora vamos a centrarnos en la definición básica, que requiere que tenga lugar la transferencia de una persona -coercitiva, engañosa o abusiva- con intención de explotarla, pero sin que sea necesario que se materialice la explotación (Chuang 2014, 628-629; Stoyanova, 2017, 33-43).

Es preciso llamar la atención sobre el elemento «propósito de explotación». En la práctica, el Protocolo establece un umbral mínimo de protección, por encima del cual admite la posibilidad de ampliar el concepto para incluir una infinidad de prácticas que dependerán de la decisión de cada Estado (Pérez Alonso 2008, 181). En consecuencia, a cada Estado parte le corresponde decidir, por un lado, la medida en que adoptará disposiciones destinadas a prevenir, reprimir y sancionar la trata de personas, y por otro, la forma en que definirá la «trata de personas» dentro de su propia jurisdicción nacional y respetando ciertos márgenes (Allain 2014, 116; Rijken 2013, 13).

b. Incorporación del delito de trata en las legislaciones nacionales: ¿estamos hablando de lo mismo?

La situación anterior ha provocado que los Estados hayan incorporado la definición de trata de forma muy diversa, como demuestra el hecho que algunas legislaciones nacionales prescindan de la utilización de unos determinados medios comisivos (coacción, engaño, amenazas, etc.) en el tipo básico. Por ejemplo, el artículo 2 de la Modern Slavery Act de 2015, para Inglaterra y Gales, establece que «Una persona comete un delito si organiza o facilita el viaje de otra persona (V) con miras a la explotación de $V »$, con independencia del consentimiento de $\mathrm{V}$ y sin que tenga que certificarse el recurso a la violencia, amenazas, engaño o abuso de situación de necesidad.

De una forma idéntica lo regula Irlanda del Norte (artículo 2 de la Human Trafficking and Exploitation (Criminal Justice and Support for Victims) Act de 2015) y Escocia (artículo 1 de la Human Trafficking And Exploitation Act, de 2015). En ese mismo sentido, Argentina castiga con una pena de cuatro a ocho años a aquel que «ofreciere, captare, trasladare, recibiere o acogiere personas con fines de explotación, ya sea dentro del territorio nacional, como desde o hacia otros países, aunque mediare el consentimiento de la víctima» (artículo 145 bis del Código Penal). En este caso, el empleo de los medios comisivos a los que se refiere el Protocolo de Palermo actúa como circunstancia agravante (artículo 145 ter). Podemos nombrar otros ejemplos similares en Eslovenia (artículo 113 del Código Penal), Ecuador (artículo 91 del Código Penal), Hungría (artículo 192 del Código Penal), México (artículo 10 del Código Penal), Uruguay (artículo 78 y 79 de la Ley No. 18250 sobre migraciones), o Filipinas (artículo 4 de la Ley No. 9208), entre otros. 
Por el contrario, las normativas nacionales han tendido a prestar mucha atención al último de los elementos de la definición del Protocolo: el elemento "propósito de explotación», incluyendo minuciosas y extensas listas de prácticas que pretenden ampliar el ámbito de protección respecto al mínimo marcado en el Protocolo de Palermo. Aunque un estudio detallado de cómo se ha transpuesto la definición de trata en las legislaciones nacionales excede el ámbito de este trabajo, vamos a ilustrarlo con algunos ejemplos.

El StGB alemán prohíbe la trata de seres humanos (Menschenhandel) en el artículo 232, siguiendo la estructura de acción, medios comisivos (aprovechamiento de la situación personal o económica de la víctima, o de su impotencia en relación con su estancia en un país extraño), si se realiza con el objetivo de explotarla: 1. (a) En el ejercicio de la prostitución o en la realización de actos sexuales sobre o delante del delincuente o de una tercera persona o en la aquiescencia de actos sexuales contra uno mismo por parte del delincuente o de una tercera persona; (b) en el empleo; ${ }^{1}$ (c) en el ejercicio de la mendicidad; o (d) en la comisión de actos sancionados con una pena; 2 . Si se mantiene a la persona en esclavitud, servidumbre, servidumbre por deudas o en circunstancias análogas o similares a la esclavitud; o 3. Si se le extrae ilegalmente algún órgano (Spitzer 2018).

Por otro lado, el Código Penal francés prohíbe la trata de personas (traite des êtres humains) en el artículo 225-4-1 de una forma similar: acción, medios comisivos y propósito de explotación, que incluye: proxenetismo, agresión o abuso sexual, esclavitud, trabajos o servicios forzados, servidumbre, extracción de órganos, explotación de la mendicidad, la imposición de condiciones de trabajo o de alojamiento que sean contrarias a la dignidad humana, $u$ obligar a la víctima a cometer un delito o una falta (Bourgeois 2017).

Las diferencias se acentúan si nos alejamos de los Estados de la Unión Europea a los que les ha repercutido la normativa europea sobre trata (Pérez Alonso 2015, 1147-1194, Villacampa 2011, 203-229) y el mecanismo de la orden europea de detención y entrega (Satger 2019, 45-46). Así, los Estados han establecido como prácticas abusivas conductas muy singulares. Filipinas, por ejemplo, establece una prolija lista de posibles propósitos de explotación, entre los que incluye la prostitución voluntaria o el turismo sexual (párrafos (e) y (d) del artículo 4 de la Ley No. 9208, respectivamente); Moldavia incluye «el uso de una mujer como madre subrogada o para otros propósitos reproductivos» (artículo 2.3 de Ley para Prevenir y Combatir la Trata

${ }^{1}$ En el propio artículo especifica que se considera que existe explotación «en el empleo» si se impone con el fin de obtener un beneficio implacable en condiciones de trabajo que sean manifiestamente desproporcionadas con respecto a las condiciones de trabajo de dichos empleados que se dedican a la misma ocupación o a una ocupación comparable. 
de Seres humanos, de 2005); Bulgaria se refiere, entre otras finalidades, al «libertinaje» (artículo 159a del Código Penal); el artículo 182 del Código Penal nicaragüense a la «experimentación biomédica clínica o farmacológica ilícita»; Uruguay a la realización de «cualquier otra actividad que menoscabe la dignidad humana» (artículo 78 de la Ley No. 18250 sobre migraciones), y el Código Penal boliviano se refiere al «matrimonio servil» o al «turismo sexual» (artículo 281 bis), entre otras finalidades de una larga lista. ${ }^{2}$

En definitiva, a pesar de los avances hacia una armonización penal que allanan el camino de la cooperación interestatal, el amplio margen de discrecionalidad de los Estados no ha impedido, en la práctica, la subsistencia de un régimen fragmentado en la lucha contra la trata. En otras palabras: a pesar de tratarse de una definición fijada a nivel internacional, no puede olvidarse que su verdadera aplicación tiene lugar a nivel nacional, donde los Estados parte del Protocolo de Palermo han ofrecido lecturas muy singulares del delito de trata.

\section{c. La fragmentación del régimen contra la trata: ¿cuáles son las consecuencias?}

El Protocolo de Palermo crea únicamente un marco de actuación que permite y facilita la cooperación interestatal, porque es un instrumento de naturaleza transnacional que se diferencia de los instrumentos internacionales en que no existe una autoridad central de naturaleza internacional que controle su aplicación, sino que funciona de manera puramente anárquica (Kobrin 2008, 252; Boister 2012). En este contexto, la armonización de las definiciones desempeña un papel fundamental, puesto que la fragmentación dificulta la consecución del objetivo de asistencia mutua en los asuntos penales y afecta a la eficacia del régimen transnacional.

Uno de los aspectos principales en el objetivo de asistencia mutua en asuntos penales es la extradición, donde entra en juego el principio de la doble tipificación de los hechos: ${ }^{3}$ no basta con que los crímenes tengan el

2 1. Venta u otros actos de disposición del ser humano con o sin fines de lucro. 2. Extracción, venta o disposición ilícita de fluidos o líquidos corporales, células, órganos o tejidos humanos. 3. Reducción a esclavitud o estado análogo. 4. Explotación laboral, trabajo forzoso o cualquier forma de servidumbre. 5. Servidumbre costumbrista. 6. Explotación sexual comercial. 7. Embarazo forzado. 8. Turismo sexual. 9. Guarda o adopción. 10. Mendicidad forzada. 11. Matrimonio servil, unión libre o de hecho servil. 12. Reclutamiento de personas para su participación en conflictos armados o sectas religiosas. 13. Empleo en actividades delictivas. 14. Realización ilícita de investigaciones biomédicas.

${ }^{3}$ Es preciso señalar que el principio de la doble tipificación en el contexto de la trata tiene relevancia fuera del contexto de la Unión Europea. Esto se debe a que la Decisión Marco 2002/584/JAI del Consejo, de 13 de junio de 2002, relativa a la orden de detención europea y a los procedimientos de entrega entre Estados miembros en los países (Euroor- 
mismo nombre o que ambos Estados sean parte del Protocolo Palermo, sino que se requiere una cierta simetría entre el derecho interno del Estado que solicita la extradición y del Estado requerido respecto al delito en concreto (Williams 1991, 581-583; Edmonds-Poli y Shirk 2018, 216-220). El principio de doble tipificación, dirigido a proteger la soberanía de los Estados (Blakesley 2000,4), se basa en una caracterización recíproca de los delitos y en una especie de mutualidad de las obligaciones entre Estados (Bassiouni 1974, 314).

Por lo tanto, el juez nacional tendrá que asegurarse de que, de hecho y de derecho, lo que se denomina trata en otro país también se corresponde con la caracterización de la trata de personas en su jurisdicción. En este contexto, el hecho de que los Estados hayan incorporado en su derecho interno distintas versiones del delito de trata de personas y los elementos que lo componen dificulta la creación de un régimen transnacional. Por ejemplo, en Filipinas se castiga la conducta de captar, transportar, recibir, etc., con cualquier medio, con fines de prostitución. ${ }^{4}$ No obstante, hay países, como Holanda, donde la prostitución voluntaria es legal. En este caso, si Filipinas quiere procesar a los miembros de una red de trata que afecta a su ámbito territorial -de acuerdo con su propia definición del delito- pero que se encuentran en Holanda, en principio no sería posible la extradición.

\section{d. La trata desde el paradigma de la explotación}

El argumento que pretendo desarrollar parte de que, en lugar de poner el foco en los distintos tipos de explotación que pueden estar englobados en el elemento «intención o propósito de explotación», es preciso centrarse en qué es lo que convierte la trata en una práctica abusiva o «de explotación» por sí misma. Si bien es cierto que suele ser el paso previo a la explotación, no se trata simplemente de un proceso inocuo o «un delito de peligro respecto del de explotación subsiguiente» (Lloria García 2019, 370). ¿Pero por qué?

Con carácter preliminar, es preciso señalar que la afirmación de que la trata es una práctica de explotación no supone admitir que la trata equivale a sus finalidades. Algunos autores han identificado la trata con la esclavitud o el trabajo forzoso (Koettl 2009, 6; Gallagher 2009, 814; Gallagher 2010, 30-31; Roth 2012, 73), basándose en el carácter amplio de las acciones del

den) garantiza la extradición y entrega, sin control de la doble tipificación, en los casos de trata de seres humanos (artículo 2.2). Ver: Bachmaier 2016.

${ }^{4}$ Artículo 4(a) de la Ley No. 9208, reformada por la Ley No. 10364, sobre trata de personas: «Captar, obtener, contratar, proporcionar, ofrecer, transportar, transferir, mantener, albergar o recibir a una persona por cualquier medio, incluidos los realizados con el pretexto de empleo o formación o aprendizaje en el país o en el extranjero, con fines de prostitución, pornografía o explotación sexual. 
delito de trata, especialmente la «recepción». No obstante, pueden esgrimirse cuatro motivos que nos llevan a rechazar esta interpretación: en primer lugar, el más evidente, por el sentido literal de los términos de la definición de trata de personas, que indican expresamente que el traslado debe producirse con la finalidad de explotación (elemento subjetivo del injusto), sin que se tenga que materializar (Obokata 2006, 20). ${ }^{5}$ En segundo lugar, porque iría en contra del sentido de los propios travaux prèparatoires del Protocolo de Palermo, de donde se deduce que en la trata de seres humanos debe existir algún tipo de movimiento, la preparación de este o la recepción de las personas después del movimiento (Chuang 2014, 630-631). En tercer lugar, porque la convergencia completa entre la trata y la subsiguiente explotación sería contraria al propósito del resto de tratados que prohíben la explotación, entre los que se inserta este Protocolo. Estos tratados, que se crean durante el siglo XX para prohibir la esclavitud y otras formas de explotación, nunca pretendieron abarcar las prácticas que actualmente se asocian con la trata de personas y que tenían un régimen aparte. Por último, porque se vaciaría de contenido práctico al delito de trata y sería posible calificar como trata un gran número de situaciones, como los casos de violación o abuso sexual (Stoyanova 2017, 38).

Partiendo de estas consideraciones, es preciso recordar que no existe un concepto unificado de explotación a nivel internacional (Rijken 2013, 12). En la mayoría de los casos, la naturaleza abusiva o «de explotación» de la relación va a venir determinada por el incumplimiento de la normativa laboral estatal (Lousada Arochena 2018, 158-159), que proporciona seguridad jurídica sobre los contornos de las conductas permitidas y las que no. No obstante, a la hora de valorar qué condiciones hacen que, en general, un comportamiento sea «de explotación», Jean Allain propone utilizar el marco teórico elaborado por Alan Wertheimer en su obra Exploitation (Wertheimer 1996; Wertheimer 1997; Zwolinski, y Wertheimer 2016), que continúa siendo el estudio más profundo sobre los contornos de las conductas de explotación (Allain 2019, 5).

El estudio de la explotación ha sido un tema recurrente en la literatura científica, especialmente en economía y sociología, y se ha tendido a prestar mayor atención al estudio de las condiciones generales que hacen que una sociedad sea «justa», respondiendo fundamentalmente a preguntas sobre cómo deben distribuirse los recursos escasos o cuáles son las libertades o derechos básicos (Wertheimer 1996, 8). No obstante, el hecho de que una

${ }^{5}$ Como indica la Convención de Viena sobre el derecho de los tratados, «un tratado deberá interpretarse de buena fe, conforme al sentido corriente que haya de atribuirse a los términos del tratado, en el contexto de estos, y teniendo en cuenta su objeto y fin» (artículo 31$)$. 
sociedad tenga las condiciones adecuadas para que puedan producirse transacciones justas, no impide que también pueda existir explotación a nivel particular (Shklar, 1989, 1135; Barry, 1989, 305).

Esta explotación a nivel micro es la que examina Wertheimer, y concretamente qué condiciones intervienen para que una conducta sea abusiva o de explotación. De las principales definiciones normativas de explotación que ponen el énfasis en distintos aspectos, como la instrumentalización del explotado, la coacción, el daño o el refuerzo de la inequidad entre las partes (Tormey 1974, 207-208; Munzer, 1990, 171; Buchanan 1985, 87; Sample 2003, cap. 4; Feinberg 176-179; Zwolinski 2012, 154-179), Wertheimer extrae el mínimo común múltiple de todos ellos:

A explota de forma perjudicial/abusiva a B cuando obtiene una ventaja injusta de B (Wertheimer 1996, 16).

Con esta definición, la clave para saber cuándo la explotación es abusiva residirá en la determinación de que la ventaja que obtiene A es injusta. ¿Y cuándo es injusta? Wertheimer señalas las siguientes categorías para determinarlo:

a) Defecto en el proceso: una transacción será injusta si A utiliza o crea un defecto en el proceso de la transacción con $\mathrm{B}$ que le beneficie a expensas de B (Wertheimer 1996, 25-28). Este defecto en el proceso puede producirse cuando existe coacción o engaño, o cuando B tiene unas condiciones de partida injustas o desesperadas que le impiden consentir de una forma válida. Por ejemplo, si $\mathrm{A}$ amenaza con un daño a $\mathrm{B}$ o a alguien de su familia para que acepte, o si A se aprovecha de una situación -o defecto- preexistente, aunque no exista coacción o engaño.

b) Efecto en los intereses de B: esto se produce, a grandes rasgos, cuando la compensación de $\mathrm{B}$ es demasiado baja o se trata de una tarea que se considera objetivamente degradante (Wertheimer 1997, 898). En definitiva, cuando la situación de $\mathrm{B}$ empeora notablemente en relación con lo que tenía antes de relacionarse con A y como consecuencia de dicha relación.

Vamos a centrarnos en el primer aspecto. En una situación de explotación, $\mathrm{A}$ obtiene un beneficio que daña $\mathrm{a} \mathrm{B}$, aprovechándose normalmente de una situación de vulnerabilidad personal, derivada de sus características físicas, o de otros factores. En la conceptualización de la trata de personas, el Protocolo de Palermo no pone el foco en el beneficio que obtiene A, sino en elproceso de traslado de B que se realiza de una determinada manera: mediante amenazas, uso de la fuerza u otras formas de coacción, rapto, fraude, engaño, abuso de poder o de una situación de vulnerabilidad, etc.

Estos «defectos en el proceso» llevan a un resultado injusto, que es la capacidad de aprovecharse de B de una manera abusiva. No obstante, si bien 
está claro que la coacción, el engaño o las amenazas convierten la trata en un fenómeno abusivo, el siguiente paso lógico será plantearse cuál es el umbral a partir del cual el abuso de una situación de vulnerabilidad es relevante. ¿Basta con que exista una situación de vulnerabilidad objetiva para que sea operativa en el contexto de la trata?

El paradigma de la explotación obliga a que debamos adoptar un enfoque restrictivo del concepto de abuso de situación de vulnerabilidad que sea equiparable a la coacción, engaño o amenazas en su capacidad de provocar un «defecto en el proceso» que lleve a un resultado injusto. Es decir, que no es suficiente con probar que hay una situación de vulnerabilidad de forma objetiva, de naturaleza lo suficientemente grave, sino que es preciso demostrar que se ha producido un abuso de esta, y que ese abuso ha sido relevante y suficiente para situar a la persona en el contexto de la trata, al menos de una forma equiparable al resto de medios (UNODC 2016, 75-76).

La naturaleza de las condiciones impuestas en el país de recepción puede ayudar a evaluar la existencia de un abuso específico de una situación de vulnerabilidad, puesto aunque se alegue que la persona conocía que en el Estado receptor iba a dedicarse, por ejemplo, a la prostitución, un resultado claramente contrario a los intereses de B -porque las condiciones impuestas sean manifiestamente inferiores a los estándares de la normativa laboral del paísconstituye un indicio de que ha existido un «defecto en el proceso», y por ende, trata de personas.

Por último, el foco en la naturaleza abusiva o de explotación per se de la trata de personas también nos permite evaluar críticamente aquellas legislaciones que omiten el elemento de la utilización de los medios comisivos para la configuración del tipo básico de trata, así como las afirmaciones que ponen en tela de juicio la importancia de la distinción entre la trata y el tráfico ilícito de migrantes (Salt and Stein 1997; Salt 2000. Campana y Varese realizan una descripción crítica de estos planteamientos en: Campana y Varese 2016, 90-92). La eliminación de los medios comisivos de la estructura del delito de trata distorsiona la naturaleza autónoma del fenómeno de trata y difumina las fronteras con la inmigración ilegal y, en definitiva, dificulta el establecimiento de un régimen efectivo de cooperación transnacional.

\section{CONCLUSIÓN}

Tras el transcurso de casi dos décadas desde la aprobación de la primera norma transnacional que unifica la lucha contra la trata de personas, podemos evaluar críticamente si se ha consolidado un régimen transnacional que permita la asistencia mutua eficaz en materia penal o si, por el contrario, continúa tratándose de un régimen fragmentado. A pesar de la armonización del concepto de trata, una defectuosa comprensión de la naturaleza abusiva de la trata 
ha dado lugar a lecturas muy singulares del fenómeno que se ha reflejado en la normativa doméstica. En este sentido, algunos Estados han incorporado un delito de trata prescindiendo de los medios comisivos en la estructura típica -es decir, sin que tenga que recurrirse a la coacción, engaño o abuso de situación de vulnerabilidad - (por ejemplo, Reino Unido), o han incorporado prolijas listas de lo que se considera «explotación» a efectos de trata, incluyendo prácticas como la prostitución voluntaria (por ejemplo, Filipinas).

Las distintas lecturas de la naturaleza de la trata de personas pueden tener su reflejo en la eficacia del régimen transnacional respecto a instituciones de cooperación interestatal como la extradición, donde se requiere que exista una cierta simetría entre el derecho interno del Estado que solicita la extradición y del Estado requerido en relación al delito en concreto (principio de doble tipificación): no es suficiente con que los delitos tengan el mismo nombre o que ambos Estados sean parte del Protocolo Palermo.

Una reformulación del paradigma de la trata puede ser útil para acometer los desafíos que plantea la creación y el mantenimiento de cualquier régimen transnacional. Así, el principal argumento de este trabajo reside en que, en lugar de poner el foco en los distintos tipos de explotación que pueden estar englobados en el elemento «intención o propósito de explotación», es preciso centrarse en qué es lo que convierte la trata en una práctica abusiva o «de explotación» por sí misma. La conceptualización de la explotación como una forma injusta de aprovechamiento de otra persona (Wertheimer 1996) y el reconocimiento de que en la trata esto se manifiesta a través los medios comisivos que obligan al movimiento, con la finalidad de colocar a la persona en una situación en la que puede ser explotada, permite forjar un espacio legal claro para la trata. Es decir, lo que convierte la trata en una conducta de explotación es la existencia de un «defecto en el proceso» que debe tener una entidad suficiente, y donde las condiciones finalmente impuestas son indicadores de la existencia de dicho defecto.

Este enfoque también nos ayuda a identificar las conductas relevantes en términos de explotación y permite abordar las críticas de los que -en ocasiones, acertadamente- ven en el régimen contra la trata una herramienta de control migratorio más. En definitiva, este trabajo continúa en la línea de reflexión de un fenómeno complejo y multifacético como la trata de personas, y está dirigido a promover la tarea de asistencia mutua en asuntos penales y transfronterizos y a captar la esencia de la trata como una conducta de explotación.

\section{BIBLIOGRAFÍA}

Allain, Jean. 2019. «Conceptualizing the exploitation of human trafficking». En The SAGE handbook of Human Trafficking and Modern Day Slavery, editado por Jennifer Bryson Clark y Sasha Poucki, 3-17. Londres: SAGE Publications Ltd. 
2014. «No Effective Trafficking Definition Exists: Domestic Implementation of the Palermo Protocol». Albany Government Law Review 7, nº. 1: 111-142

AtAK, Idil y James C. SimEON. 2014. «Human Trafficking. Mapping the Legal Boundaries of International Refugee Law and Criminal Justice». Journal of International Criminal Justice 12, no. 5: 1019-1038.

Bachmaier, Lorena. 2016. Tratado de Derecho y Politicas de la Unión Europea (Tomo VIII). Pamplona: Editorial Aranzadi.

BARRY, Brian. 1989. Theories of Justice. Berkeley: University of California Press.

Blakesley, Christopher L. 2000. «Autumn of the Patriarch: The Pinochet Extradition Debacle and Beyond-Human Rights Clauses Compared to Traditional Derivative Protections Such as Double Criminality». Journal of Criminal Law and Criminology 91, nº. 1: 1-98

Boister, NeIL. 2012. An Introduction to Transnational Criminal Law. Oxford: Oxford University Press.

Bourgeols, Statutory Progress and Obstacles to Achieving an Effective Criminal Legislation against the Modern-Day Forms of Slavery: The Case of France, 38 Mich. J. Int'l L. 455 (2017).

Buchanan, Allen. 1985. Ethics, Efficiency and the Market. Totowa: Rowman and Alanheld.

Campana, Paolo y Federico VArese. 2016. «Exploitation in Human Trafficking and Smuggling». European Journal of Criminal Policy and Research 22: 89-105.

ChuAng, Janie A. 2014. «Exploitation Creep and the Unmaking of Human Trafficking Law». American Journal of International Law 108: 609-649.

De León Villalba, Francisco Javier. 2003. Tráfico de personas e inmigración ilegal. Valencia: Tirant lo Blanch.

DoezemA, Jo. 1998. «Forced to Choose: Beyond the Voluntary v. Forced Prostitution Dichotomy». En Global Sex Workers: Rights, Resistance and Redefinition, editado por Kamala Kempadoo y Jo Doezema, 34-50. Nueva York: Routledge.

Gallagher, Anne. 2010. The International Law of Human Trafficking. Cambridge: Cambridge University Press.

— 2009. «Human Rights and Human Trafficking: Quagmire or Firm Ground? A Response to James Hathaway». Virginia Journal of International Law 49, nº 4 : 789.

GARCÍA ARÁN, Mercedes. 2006. «Introducción». En Trata de personas y explotación sexual, coordinado por Mercedes García Arán. Granada: Comares

GrITTNER, Frederik K. 1990. White Slavery: Myth, Ideology and American Law. New York: Garland

HeInZTe, Hans-Joachim y Charlotte LÜLF. 2016. «The UN Protocol to Prevent, Suppress, and Punish Trafficking». En International Law and Transnational Organised Crime, editado por Pierre Hauck, Pierre y Sven Peterke. Oxford: Oxford University Press: 150-168.

KeLley, Judith G. 2017. Scorecard diplomacy: Grading states to influence their reputation and behavior. Cambridge: Cambridge University Press.

KEMP, Gerhard. 2001. «The United Nations Convention against Transnational Organized Crime: A Milestone in International Criminal Law». South African Journal of Criminal Justice 14, $\mathrm{n}^{\circ}$. 2: 152-167. 
KoETTL, Johannes. 2009. «Human Trafficking, Modern Day Slavery, and Economic Exploitation». SP Discussion Paper No. 0911. Social Protection \& Labor. The World Bank.

Kobrin, Stephen J. 2008. «Globalization, Transnational Corporations and the Future of Global Governance». En Handbook of Research on Global Corporate Citizenship, editado por Andreas Georg Scherer y Guido Palazzo.

Lloria García, Paz. 2019. «El delito de trata de seres humanos y la necesidad de creación de una Ley Integral». Estudios Penales y Criminológicos 39: 353-402.

López Rodríguez, Josune. 2016. Conceptualización jurídica de la trata de seres humanos con fines de explotación laboral. Navarra: Editorial Aranzadi.

LÓPEz Rodríguez, Josune y Francisco Javier ArRIETA IDIAKEZ. 2019. «La trata de seres humanos con fines de explotación laboral en la legislación española». icade. Revista cuatrimestral de las Facultades de Derecho y Ciencias Económicas y Empresariales 107: 1-24.

Maqueda Abreu, María Luisa. 2018. «Trata y esclavitud no son lo mismo, pero ¿qué son?». En Estudios jurídicos penales y criminológicos. En homenaje al Prof. Dr. H. C. Mult. Lorenzo Morillas Cueva, dirigido por José María Suárez López et al, 1251-1264. Madrid: Dykinson.

- 2009. Prostitución, feminismos y derecho penal. Granada: Comares.

MARAver Gómez, Mario. 2011. «Capítulo XII. La trata de seres humanos». En Estudios sobre las Reformas del Código Penal operadas por las LO 5/2010, de 22 de junio, y 3/2011, de 28 de enero, dirigido por Julio Díaz-Maroto y Villarejo. Navarra: Editorial Aranzadi.

Milivojevic, SANJA y Sharon Pickering. 2013. «Trafficking in People, 20 Years On: Sex, Migration and Crime in the Global Anti-Trafficking Discourse and the Rise of the Global Trafficking Complex». Current Issues in Criminal Justice 25, nº 2 : 585- 604.

Munzer, Stephen R. 1990. A Theory of Property. Cambridge: Cambridge University Press.

DAVIDSON, Julia O'Connell. 2010. «New slavery, old binaries: human trafficking and the borders of 'freedom'». Global Networks 10, n'. 2: 244-261.

Овоката, Tom. 2006. Trafficking of Human Beings from a Human Rights Perspective : Towards a Holistic Approach. Leiden: Martinus Nijhoff Publishers.

PÉrez Alonso, Esteban. 2015. «La política europea en materia de trata de seres humanos». Revista de la Facultad de Derecho de la Universidad de Granada, $\mathrm{n}^{\circ}$. 16-18: 1147-1194.

— 2008. Tráfico de personas e inmigración clandestina (Un estudio sociológico, internacional y jurídico-penal). Valencia: Tirant lo Blanch.

PÉREZ CEPEDA, Ana Isabel. 2004. Globalización, tráfico internacional ilicito de personas y derecho penal. Granada: Comares.

Pomares CinTAS, Esther. 2013. El derecho penal ante la explotación laboral y otras formas de violencia en el trabajo. Valencia: Tirant lo Blanch.

— 2011. «El delito de trata de seres humanos con finalidad de explotación laboral». Revista Electrónica de Ciencia Penal y Criminología 13-15: 1-31. 
RIJKEN, Conny. 2013. «Trafficking in Human Beings for Labour Exploitation: Cooperation in an Integrated Approach». European Journal of Crime, Criminal Law and Criminal Justice 21, no. 1: 9-36

Roth, Venla. 2012. Defining Human Trafficking and Identifying Its Victims: A Study on the Impact and Future Challenges of International, European and Finnish Legal Responses to Prostitution-Related Trafficking in Human Beings. Leiden: Martinus Nijhoff Publishers.

SÁnchEZ-CovisA VILlA, Joaquín. 2016. «El delito de trata de seres humanos. Análisis del artículo 177 bis CPc. Cuadernos de la Guardia Civil, Revista de Seguridad Pública 52: 36-51.

SATGER, Helmut. 2019. «Is mutual recognition a viable general path for cooperation?». New Journal of European Criminal Law 10, nº. 1: 44-56

SCARPA, Silvia. 2008. Trafficking in Human Beings: Modern Slavery. Oxford: Oxford University Press.

SALT, John. 2000. «Trafficking and human smuggling: a European perspective». International Migration 38, $\mathrm{n}^{\circ} .3$ : 31-56.

SALT, John y Jeremy STEIN. 1997. «Migration as a business: the case of trafficking». International Migration 35, $\mathrm{n}^{\mathrm{o}}$. 4: 467-494.

ShKLAR, Judith. 1989. «Giving Injustice Its Due». Yale Law Journal 98: 1125.

SPITZER, Ana-Lenna. 2018. Strafbarkeit des Menschenhandels zur Ausbeutung der Arbeitskraft. Nueva York: Springer.

Stoyanova, Vladislava. 2017. Human Trafficking and Slavery Reconsidered. Conceptual Limits and States' Positive Obligations in European Law. Cambridge: Cambridge University Press.

TAYLOR, Julie L. y Kirsten L. IsGRO. 2018. «TIP Reports as Policy and Narrative: Exploring the Communicative Framework for Policy Enactment». Journal of Human Trafficking 4, $\mathrm{n}^{\circ}$. 1: 48-60.

TORMEY, Judith Farr. 1974. «Exploitation, Oppression and Self-Sacrifice». Philosophical Forum 5: 206-221.

UNODC. 2016. Poner referencia https://www.unodc.org/documents/human-trafficking/2016/Abuse_of_a_position_of_vulnerability_Spanish.pdf de 2016

VillacAmpa Estiarte, Carolina. 2011. El delito de trata de seres humanos. Una incriminación dictada desde el Derecho Internacional. Navarra: Editorial Aranzadi.

2011a. «La nueva Directiva Europea relativa a la prevención y lucha contra la trata de seres humanos y a la protección de las víctimas. ¿cambio de rumbo de la política de la Unión en materia de trata de seres humanos?». Revista Electrónica de Ciencia Penal y Criminología 13-14: 1-52.

WertheIMER, Alan. 1997. «Remarks on Coercion and Exploitation». Denver University Law Review 74: 889.

— 1996. Exploitation. New Jersey: Princeton University Press.

WiLliams, Sharon A. 1991. «The Double Criminality Rule and Extradition: A Comparative Analysis». Nova Law Review 15, nº. 2: 581-624

ZwOLInSKI, Matt y Alan WertheIMER. 2016. «Exploitation». En The Stanford Encyclopedia of Philosophy, editado por Edward N. Zalta. 


\title{
REEXAMINANDO LA DEFINICIÓN DE TRATA DE SERES HUMANOS DEL PROTOCOLO DE PALERMO: LA TRATA COMO FORMA DE EXPLOTACIÓN
}

\author{
Unwrapping the Human Trafficking Definition of the \\ Palermo Protocol: Trafficking as Harmful Exploitation
}

\author{
Ana B. Valverde Cano \\ Doctoranda en Derecho penal \\ Universidad de Granada
}

http://dx.doi.org/10.18543/ed-67(2)-2019pp15-29

\section{Copyright}

Estudios de Deusto es una revista de acceso abierto, lo que significa que es de libre acceso en su in tegridad. Se permite su lectura, la búsqueda, descarga, distribución y reutilización legal en cualquier tipo de soporte sólo para fines no comerciales, sin la previa autorización del editor o el autor, siempre que la obra original sea debidamente citada y cualquier cambio en el original esté claramente indicado

Estudios de Deusto is an Open Access journal which means that it is free for full access, reading, search, download, distribution, and lawful reuse in any medium only for non-commercial purposes, without prior permission from the Publisher or the author; provided the original work is properly cited and any changes to the original are clearly indicated. 